\title{
BMJ Open Does changed referral options affect the use of MRI for patients with low back pain? Evidence from a natural experiment using nationwide data
}

\author{
Morten Sall Jensen, ${ }^{1,2}$ Kim Rose Olsen, ${ }^{3}$ Lars Mors $\varnothing,{ }^{1,4}$ Jens Søndergaard, ${ }^{5}$ \\ Berit Schiøttz-Christensen ${ }^{1,6}$
}

To cite: Jensen MS, Olsen KR, Mors $\emptyset$ L, et al. Does changed referral options affect the use of MRI for patients with low back pain? Evidence from a natural experiment using nationwide data. BMJ Open 2019;9:e025921. doi:10.1136/ bmjopen-2018-025921

- Prepublication history and additional material for this paper are available online. To view these files, please visit the journal online (http://dx.doi org/10.1136/bmjopen-2018025921).

Received 14 August 2018 Revised 30 May 2019 Accepted 7 June 2019

\section{Check for updates}

(c) Author(s) (or their employer(s)) 2019. Re-use permitted under CC BY-NC. No commercial re-use. See rights and permissions. Published by BMJ.

For numbered affiliations see end of article.

Correspondence to Morten Sall Jensen; mosj@vive.dk,

morten_sall@hotmail.com

\section{ABSTRACT}

Objectives This study reports lumbar MRI referral patterns in the Region of Southern Denmark (RSD) and investigates the hypothesis that we will see an increase in imaging rates (MRI rates) following new referral options to lumbar MRI in the RSD in comparison with the other regions in Denmark from 2010 to 2013.

Design A difference-in-difference (DD) analysis, using general practitioners (GPs) in other regions as control, was used to test if the new referral options had an effect on the MRI rates. Setting In 2010, RSD introduced organisational changes affecting the referral options for lumbar MRI. First, the possibility for direct referral to lumbar MRI was introduced GPs, and second, the region gathered all local spine departments into one specialist hospital called the Spine Centre.

Participants We retrieved all lumbar MRIs performed on patients aged 18+ performed on Danish hospitals from 2008 to 2013 using the registries from Statistics Denmark. We use sociodemographic information from all Danish citizens aged 18+ aggregated to GP level. Primary and secondary outcome measures: lumbar MRI scans per 1000 capita enlisted with a GP (MRI rates) were calculated based on GPs patient list. Four referral types were made to describe changes in referral patterns.

Results In total 183389 patients received 240760 lumbar MRIs in the period. The use of the direct referral option by GPs in the RSD increased by $115 \%$ in the period from 2010 to 2013 and accounted for $34 \%$ of all referrals $(n=6545)$ in 2013. MRI rates were significantly higher in RSD following the organisational changes (DD 1.389(0.925-1.852) lumbar MRI per 1.000 enlisted with a GP).

Conclusions Introduction of organisational changes in RSD as direct referral to lumbar MRI from GPs and chiropractors as well as establishing a Spine Centre increase the lumbar MRI rate in comparison with other regions in Denmark.

\section{BACKGROUND}

The number of lumbar MRI undertaken in the (US Medicare population increased substantially from 1994 to 2006, despite guidelines which discourages routine use of
Strengths and limitations of this study

Nationwide registry data including sociodemographic information on all citizens aged 18+.

- Use of a difference-in-difference design for possible causal inference.

- The study might underestimate the lumbar MRIs from private hospitals.

MRI. ${ }^{1-3}$ It was estimated that the use of MRI and other imaging modalities accounted for $7 \%$ of the direct treatment costs of low back pain (LBP) in $1998 .^{4}$

The factors associated with the increased use of MRI in the diagnostics of LBP have been investigated. Research shows that the substantial geographic differences in use of spinal MRI across states in the $\mathrm{USA}^{5-8}$ can be explained by differences in local clinical practices, ${ }^{6}$ physician ownership of specialty hospitals, ${ }^{9}$ fee-for-service schemes, ${ }^{10}$ MRI scanner availability $^{11}$ and state median income per capita. ${ }^{12}$ However, these studies were undertaken in a US setting among populations that had limited access to healthcare providers and where a fee-for service incentive affected doctors' wages. Consequently, more studies are needed on factors impacting the use of lumbar MRI, in other healthcare settings. ${ }^{13-15}$

In this study, we have the opportunity of using nationwide data and hence evaluating a natural experiment. In 2010, Region of Southern Denmark (RSD) made two organisational changes.

These included, centralisation of regional spine specialist departments across regional hospitals in one spine specialist hospital. Further, general practitioners (GPs) and chiropractors (CP) were given the possibility to directly refer patients with LBP for lumbar MRI without prior referral to the Spine Centre or to office-based specialist doctors. 
In the support of the organisational changes, RSD implemented a LBP Disease Management Programme (DMP). The centralisation of the hospital occurred in the beginning of 2010, and the hospital were fully operational in mid-2010. The direct referral access was available for the GPs in the first months of 2010. Hence, we expect effects of the reform to be modest in 2010 and to increase in the following years. These changes were unique in Denmark as two of four other regions maintained decentralised spine departments and did not allow for direct referral. To date, the effect of these organisational changes in RSD have not been investigated.

The study investigates the effect of the organisational changes on use of lumbar MRI in the diagnostics of patients with LBP in RSD. Primary outcome is defined as yearly lumbar MRI rates for all individuals aged 18+. As the reform increased the possibilities to refer to lumbar MRI, we hypothesised that the yearly lumbar MRI rates from 2010 to 2013 would increase significantly in RSD compared with the other regions.

\section{METHODS \\ Design}

A longitudinal register-based study covering the Danish population aged 18+ from 2008 to 2013 . The study relies on a natural experiment using RSD as the intervention group. Two regions, Zealand Region (ZR) and the North Denmark Region (NR), maintained their organisation in the study period from 2008 to 2013. Hence, these two regions can act as good indicators of the counterfactual RSD. GPs in RSD, GPs in the Capital Region of Denmark (CR) and the Central Denmark Region (CD) were given the possibility to directly refer patients with LBP for a lumbar MRI in 2010 and 2011, respectively. This allows GPs from CD to act as controls from 2008 to 2010, while GPs from CR are excluded, as they allow referrals from GPs at the same time as RSD, which is why they cannot act as good indicators of the counterfactual development in RSD if RSD had not made the organisational changes.

\section{Primary outcome}

The primary outcome is yearly lumbar MRI per 1000 enlisted with a GP.

\section{Setting}

In Denmark, five decentralised administrative regions, including 98 municipalities, manage the tax founded healthcare system. ${ }^{16}$ Each region has a public elected council and is autonomously managing secondary healthcare services. All services provided at hospitals and officebased physicians are free of charge, while services at physiotherapists and CPs involve co-payments from the patient. GPs in Denmark have a unique patient list (GP list) of citizens, to whom the GP solely provided services. The GP list size is on average 1600 patients, and $98 \%$ of all Danes are enlisted at one of the 2,200 GP clinics in Denmark. ${ }^{16}$

\section{Data sources}

The study used data from the registries at Statistics Denmark (DST), a governmental institution providing data for research purposes. ${ }^{17}$ All registries are linkable at the individual level, using the personal civil registration number (CPR number), which are given to all Danish citizens at birth. ${ }^{18} 19$ The study includes data from the following registries:

The Danish National Patient Registry (NPR), ${ }^{20} 21$ includes information on diagnosis coded according to the International Classification of Disease (ICD-10) and procedure and surgery codes (Healthcare Classification System (SKS codes)). All NPR records are unique, due to a NPR serial numbers (unique to each patient's continuum of care at a hospital) and patients' CPR numbers.

The Danish National Health Service Register for Primary Care ${ }^{22}$ includes all contacts to the primary sector healthcare providers including GPs, CP, physiotherapists and office-based specialist doctors. The GP list and GP list size were generated by combining the unique GP id with the CPR number from patients receiving most of their services from the GP id. ${ }^{23}$ GPs with patient list size less than 300 patients were deleted as they are hypothesised to be GPs either starting up or closing down the practice. Those citizens with no information of GP id in one of the study years were allocated to a hypothetical GP id generated for each region.

Danish National Prescription Registry includes information on all prescription based analgesic drugs sold at Danish primacies. ${ }^{24}$ We identified analgesic drugs according to the Anatomical Therapeutic Chemical classification (ATC) code. $^{25}$ Analgesic drugs included ATC code NN02A and NN02B; tablet cans with >100 pills of paracetamol and ibuprofen, synthetic opioids and opioids.

We further retrieved registers on income, ${ }^{26}$ education, ${ }^{27}$ job and socioeconomic status, ${ }^{28}$ civil status ${ }^{18}$ and demographics ${ }^{18}$ from DST.

\section{Definition of lumbar MRI}

This study included data for lumbar MRI (SKS code: UXME30). ${ }^{21}$ Each MRI scan performed at a public hospital is recorded in NPR. Lumbar MRIs performed on a private hospital are recorded in the NPR if they are subsidised by the government. Patients with multiple spine MRI registrations on the same NPR serial number were identified and the UXME30 code was retained for analysis. If patients showed two or more UXME30 codes for the same day only one remained.

\section{Definition of referral mode}

A referral mode variable was defined based on two variables from the NPR: referral directly from the GP (1), directly from the $\mathrm{CP}$ or initiated by private insurance (2), directly from the office-based specialist doctors (3) and from the hospital department (4). Before 2010, we observe referrals to lumbar MRI from GPs. These are recoded into hospital registrations. 


\section{Analyses strategy}

The impact on MRI rates of the well-defined organisational changes in 2010 in RSD, is analysed as a natural experiment. The change in the other regions are used as control under the assumption that the development of MRI rates in the control regions are a good indicator of how the MRI rates would have developed in RSD in the absence of the organisational changes.

We hypothesise that the two referral options (direct GP referral and referral to the Spine Centre) drives any change in use of lumbar MRI. As patients have not chosen to live in RSD based on the access to MRI, the assumption behind our analyses strategy is that we can interpret patients as randomly assigned to a GP who by construct of the natural experiment happen to have access to the organisational changes (RSD) or not (control regions). We therefore included GPs from the ZR and the NR as controls for all years in the analysis. GPs from the CD are included from 2008 to 2010 , as they had the possibility to directly refer patients with LBP for a lumbar MRI in 2011. GPs from the CR were excluded as controls.

We use a difference in difference (DD) model to analyse the effect of the reform. The DD model estimates the effect of organisational changes by assuming that the counterfactual development in the lumbar MRI rates in the treatment group (ie, RSD) could be approximated by the development in the lumbar MRI rates of the other regions. ${ }^{29}$ For the control group to match the approximation of the counterfactual development in lumbar MRI rates in RSD the model, we rely on an assumption that there was a common trend in lumbar MRI rates before the interventions. The common trend assumption was visually inspected. Furthermore, we estimated the effects of the reform year by year for 2010, 2011, 2012 and 2013 and made a placebo test by testing for an effect of the reform before it was implemented-that is, testing for an increase in lumbar MRI rates from 2008 to 2009. This placebo test is an indirect test of the common trend. The DD approach by definition control for all time constant heterogeneity between GPs in RSD and the controls but if we expect time-varying differences occurring over time we need to add covariates. Hence, second assumption behind our approach is that there were no time-varying unobservable covariates that could explain differences in selection into a referral to lumbar MRI between GPs and between regions. ${ }^{29}$ Hence, we generally assume that citizens' need for lumbar MRI are identical among regions after controlling for observable patients' characteristics and supply factors related to LBP treatment did not change over time on the regional level. A limitation of our data set is that we only have 2 years of observation before the organisational changes, which makes the validation of the common trend assumption hard to assess. As a consequence, we supplemented the DD analysis with two robustness checks. First, we made a replication of the analysis using quarterly data instead of annual data. This gives eight pretreatment observations, which allow for a better assessment of the common trend. Second,

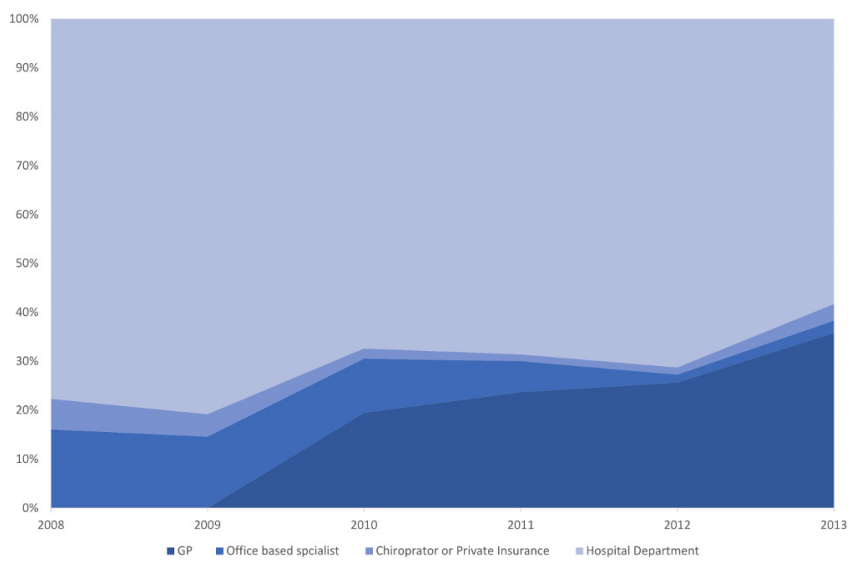

Figure 1 Changes in the referral patterns to lumbar MRI in Region of Southern Denmark from 2008 to 2013 using a $100 \%$ stacked curve diagram. GP, general practitioners.

we estimate the treatment effect using propensity score matching (PSM), an approach that does not rely on the common trend assumption but on common support. ${ }^{30-32}$

\section{Statistics}

The DD model is implemented using a parametric ordinary least squares regression model with robust SEs and clustering for GP id. We aggregated the individual level sociodemographic data to GP level. This allowed for analysis using information from the sociodemographic composition of the GPs' lists to account for any time-varying patient characteristics that is associated with LBP and therefore explain differences in GPs' referral to lumbar MRI. A supplementary advantage of using the GP as analytical level is that we in this way obtain an unbalanced panel data structure of our data set, with one observation per unique GP per year. As a robustness check, we organise data in quarterly observations and reassess the DD model using 24 quarterly observations rather than seven annual observations per GP. We further use PSM with nearest neighbour with calliper equal to one-fourth of the SD on the propensity scores. The Supplementary file 1 gives detailed information on the robustness check using quarterly data and PSM.

The changes in referral modes were graphed for RSD (see figure 1). To show dynamic year effects of the models, 2009 was used as preintervention and each intervention year was used as the postintervention year, in four DD regression models-one for each post treatment year (2010, 2011, 2012 and 2013). To test if the trend in MRI rates were not different between RSD and controls before the programme, we tested for a treatment effect in 2009 using 2008 as base year. The five models used the variables as in the adjusted models and were analysed using both control groups. The DD estimates and the $95 \%$ CI were graphed (the control regions were the $\mathrm{x}$-axis). Tables reported number $(\mathrm{N})$, means, SD, unpaired t-test and percentages (\%). All analyses were performed using STATA Release V.13 and graphics and 
Table 1 Differences in proportions of patients enlisted at a GP from either intervention or control regions for preintervention years (2008 and 2009 combined)

\begin{tabular}{|c|c|c|c|c|c|}
\hline & \multicolumn{2}{|c|}{ RSD's GPs (n=832) } & \multicolumn{2}{|c|}{ Control regions' GPs $(n=1878) \dagger$} & \multirow[b]{2}{*}{ t-testł } \\
\hline & Mean & SD & Mean & SD & \\
\hline Comorbidity score 2+ & 0.031 & 0.008 & 0.031 & 0.010 & * \\
\hline Vocational education & 0.443 & 0.029 & 0.450 & 0.038 & * \\
\hline Marital status single & 0.318 & 0.064 & 0.323 & 0.076 & * \\
\hline Gender (women) & 0.509 & 0.063 & 0.509 & 0.066 & \\
\hline $18-59$ year of age & 0.674 & 0.076 & 0.679 & 0.082 & \\
\hline GPs' id list size & 2265.060 & 3197.228 & 2212.976 & 3440.372 & \\
\hline Visits to physiotherapist & 0.086 & 0.022 & 0.096 & 0.026 & * \\
\hline Visits to chiropractor & 0.079 & 0.024 & 0.072 & 0.023 & * \\
\hline
\end{tabular}

Means reflect proportions of patients divided by the GP list size.

†Including GPs from the Zealand Region, the Central Denmark Region and the North Denmark Region.

$\ddagger$ The t-test by group with unequal variance.

${ }^{*} \mathrm{P}<0.05$.

GP, general practitioner; N, number of observations; RSD, Region of Southern Denmark.

tables were performed in Microsoft Excel 2010 (Microsoft Corporation).

\section{Covariates}

The unadjusted models included the following variables: pre-2009 and post-2009 (0=2008-2009, 1=2010-2013) and intervention and control regions $(1=\mathrm{RSD}$ and $0=\mathrm{ZR}$, NR, CD).

The adjusted models add time-varying covariates to the above variables. This is done to avoid that any observed change in RSD after the change is simply due to changes in the characteristics of the citizens over time-for example, the citizens in RSD over time becomes more prevalent to LBP than citizens in control regions. All covariates included, except GP list size, were made as proportions of enlisted patients with characteristic $\mathrm{X}$ divided with the GP list size. Patients characteristics X included; age 18-59, citizens in a full-time job, income DKK 0-399000 or missing, women, citizens living as singles and Charlson comorbidity index score ${ }^{33} 2+$, patients with vocational education, patients using a prescription on an analgesic drug at a pharmacists, patients having a visit at a physiotherapist, patients having a visit at a CP and patients visiting an office-based specialist doctor (rheumatologist, neurologist, orthopaedics and radiologist). Covariates are seen in table 1 .

\section{Patient and public involvement}

Patients were not involved in the study.

\section{Ethics}

The Danish Data Protection Agency approved this study (Journal number 15/14594). The study is based on registry data, which does not require ethics approval in Denmark (Act on Research Ethics Review of Health Research Projects $\S 14$, sec. 2 http://www.nvk.dk/ english/act-on-research 10.02.2017).

\section{RESULTS}

During the study period, 183389 patients were assessed with 240760 lumbar MRIs. Of those 27\% (63982 lumbar MRIs) were performed on private hospitals.

The figure 1 shows changes in the rates of referrals for lumbar MRI in the RSD. MRI referrals directly from GPs accounted for $18 \%(n=3044)$ of all referrals in 2010 . In the subsequent 3 years, the rate of MRI referrals directly from the GP increased to $115 \%(n=6545)$ and accounted for $34 \%$ of all of the MRI referrals in 2013. MRI referrals from office-based specialists (rheumatologist or orthopaedics) decreased from $2008(n=1916,16 \%)$ to $2013(n=464$, $2 \%)$. MRI referrals from CPs or from a private insurance decreased from $2008(n=748,6 \%)$ to $2011(n=220,1 \%)$. MRI referrals from hospital-based doctors increased from $2008(\mathrm{n}=9262,77 \%)$ to $2012(\mathrm{n}=12487,71 \%)$.

The common trend was visually inspected using figure 2, showing unadjusted average regional lumbar MRI rates for GPs in RDS and the control regions. Lumbar MRI rates for RSD increased each year starting from 14.29 lumbar MRI per 1000 enlisted with a GP in 2008 to 21.13 lumbar MRI per 1000 enlisted with a GP in 2013. The average lumbar MRI rates for three control regions increased from 7.79 lumbar MRI per 1000 enlisted with a GP in 2008 to 11.48 lumbar MRI per 1000 enlisted with a GP in 2012 and 2013. To capture any differences 


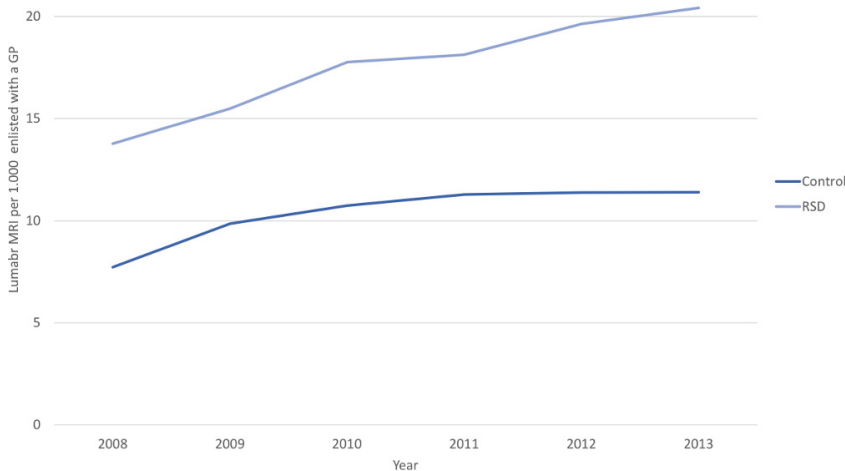

Figure 2 Lumbar MRI rates for RSD and the control regions from 2008 to 2013. GP, general practitioner; RSD, Region of Southern Denmark, Control (the Zealand Region, the Central Denmark Region and the North Denmark Region).

in time-varying trends, we included characteristics of the GP patient list, seen in table 1. The table show that there are statistical differences for 8 of the 12 included covariates between GPs in RSD and control group regions in the pre-intervention years. However, the differences are small between the patient characteristics of the GPs' lists in RSD and GPs' lists in control group regions.

The results of the DD analyses of the lumbar MRI rates per 1000 enlisted with GPs in RSD compared with GPs in the control group are shown in table 2. After the organisational changes in RSD, the lumbar MRI rates increased significantly compared with control groups, for both models ranging from 1.39 (95\% CI 0.93 to 1.85$)$ to 1.83 [95\% CI 1.37 to 2.29) lumbar MRIs per 1000 enlisted with a GP.

The table 2 also present the robustness checks using DD with quarterly data and PSM (see online supplementary table A.1 for DD results and online supplementary table A.2 for PSM results in the supplementary material). The

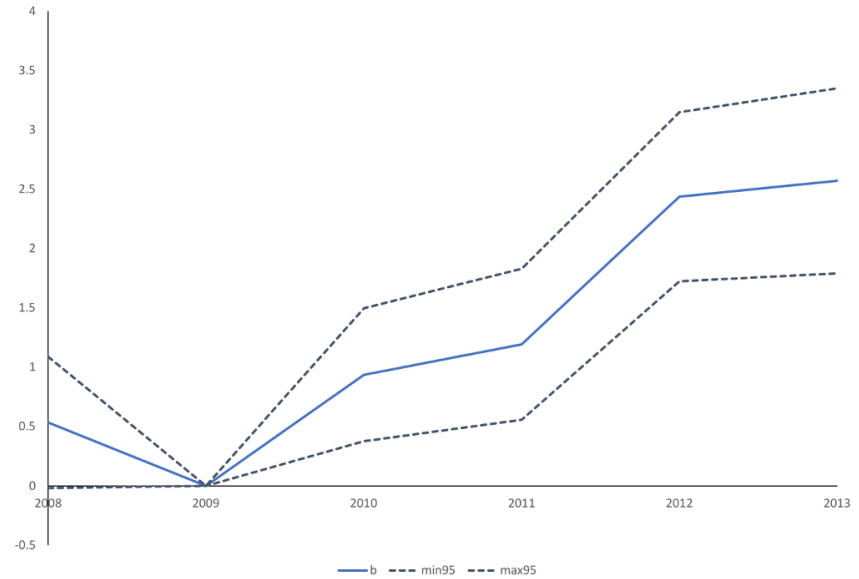

Figure 3 Dynamic year effects using the adjusted model, with multiple difference in difference estimates with 2009 as the preintervention period and all postintervention years for Region of Southern Denmark and the control regions. b, beta estimates from the difference in difference analysis; min95, lower bound of the $95 \% \mathrm{Cl}$ of the beta estimate; $\max 95$, upper bound of the $95 \% \mathrm{Cl}$ of the beta estimate.

model with quarterly data shows a significant increase in quarterly MRI of 0.43 , which is at a comparable level as the model using annual data when multiplying with four. The PSM model, however, shows quite higher effects. This may rest on the fact that RSD generally is at a higher level of MRI throughout the period of observation and that the PSM approach is less effective in taking this into account. The PSM result indicates that the DD makes a conservative estimate of the effect. We refer to the online supplementary table A3 for bias reductions of PSM model and online supplementary figure A2 for common support in supplementary material.

The dynamic year effects for both models are seen in figure 3 . The figure 3 indicates that the common trend assumption support is fulfilled, as a hypothesised treatment effect before the intervention (ie, in 2009) occurred

Table 2 DD and PSM estimates from unadjusted and adjusted models with RSD and the control regions

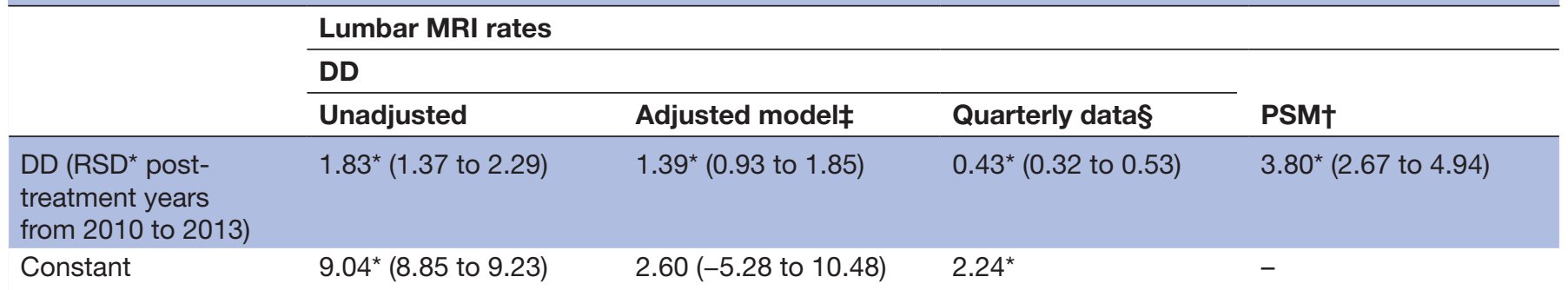

Four models showing the main outcome lumbar MRIs per 1000 enlisted with a GP.

†The PSM model use conventional options; nearest neighbour with (calliper = one-fourth of the SD on the propensity scores). We use the MRI rates in 2008 and 2009 as matching covariates to control for unobservable selection. ${ }^{31}{ }^{32}$ Furthermore, we control for clustering at GP level. ${ }^{34}$ $\ddagger$ Adjusted model include covariates for comorbidity score 2+, full time job, vocational education, marital status single, income DKK 0-399.999, male, 18-59years of age, visit at physiotherapist, visits at chiropractor, visits at office-based spine specialist, use of pain medication.

§The DD model with quarterly data use the same covariates. However, in addition, we included quarter dummies (Q1, Q2 Q3 and Q4) to take away the obvious seasonality shown in the online supplementary figure A1.

${ }^{*} \mathrm{P}<0.001$.

DD, difference in difference; GP, general practitioner; PSM, propensity score matching; RSD, Region of Southern Denmark. 
(placebo effect) is insignificant. This test is also insignificant in the model using quarterly data and hence eight pretreatment observations (see online supplementary table A1).

Dynamic year effects for postintervention years were positive and significant for all years, with an observed increase positive trend of the estimates, indicating that the effect of the organisational changes increases over time (figure 3).

\section{DISCUSSION}

This study showed that establishing a Spine Centre in the RSD and introducing direct referrals for lumbar MRI by GPs was associated with an increase in the use of lumbar MRI (compared with that of other regions) in the years following the 2010 changes. On average, the increase was between 1.39 and 1.83 lumbar MRI per 1000 enlisted with GPs in RSD involving an increase in lumbar MRI of between 1400 and 1800 additional scans compared with the other regions. The use of the direct referral option by GPs in the RSD increased by $115 \%$ in the period from 2010 to 2013 , indicating that the GP adopted the new referral option.

As in other studies from the USA, ${ }^{7}$ we find geographical variation in the use of lumbar MRI among the regions in Denmark. The reason for the difference in use of lumbar MRI among the regions is still unclear. Some points towards a special interest in back pain by specialist doctors in RSD, which relates to different regional clinical practices, which have been found in US studies. ${ }^{611}$ The relationship between MRI usages and physician incentives ${ }^{910}$ is unlikely to explain the differences in a Danish setting. First, physicians at public hospital receive a fixed yearly salary, thereby not having incentives to refer patients. Second, the public hospitals undertook $74 \%$ of all scans.

The increase in referrals for lumbar MRI following the change in GP referral access to lumbar MRI in the RSD in 2010 is noteworthy. GPs in the RSD clearly began to use the new referral option immediately and the use of the referral option increase by 115\% from 2010 to 2013. This change might indicate a lowering in the threshold for a MRI referral. There could be numerous reasons for the use of direct referrals to lumbar MRI, including patient demands ${ }^{35} 36$ and physicians' wish to provide quick reassurances to patients with $\mathrm{LBP}^{36}$ Providing quick reassurance to patients could prevent further costly visits to specialist doctors and reduce future treatment costs. However, previous studies have shown that lumbar MRI referrals from GPs are inappropriate in up to 50\% of the cases when judged against the guidelines. ${ }^{10}$ 37-39 Further, the inappropriate use of lumbar MRI has been shown to be associated with an increased use of opioids, higher healthcare costs and has a low impact on pain relief or functional recovery after 6 months in patients with non-specific LBP, without serious pathologies such as cancer, nerve root compression, cauda equina, radiculopathy and sciatica. ${ }^{35}{ }^{40-46}$ Further studies are needed to investigate if the same associations are found in this setting.

\section{Strengths and limitations}

This study used DD estimates to capture the effect of the organisational changes and LBP DMP in RSD. DD is a popular design for evaluation of policy changes, as is widely used in social science. ${ }^{29}$ DD relies on the assumption of a common trend in the pretreatment period for the outcomes of interest. This assumption seems to be fulfilled in the current study. However, a clear limitation of our data set is that we have a short pretreatment period. As a consequence, we have used DD on quarterly data as well as PSM analysis to check the robustness of the results. Both analyses support the findings and as we believe the DD approach to be the most conservative, we stick to this model as our base case. Details on the robustness model are to be found in the online Supplementary file 1.

Referrals from GPs are seen from 2008 to 2009 and are recoded to hospital referrals, as they did not have the opportunity to refer patients with LBP for a lumbar MRI. There might be several explanations for these registrations. First, registrations with referrals from GP in 2009 may be test of the electronic referral system, used in the communication of between GPs and hospitals. Second, referrals from GPs can be interpreted in relation to GPs referring patients with LBP for a consult in secondary care, where the GP refer the patient for a lumbar MRI on the same day as the consult. This allows the hospital-based specialist doctor to assess the lumbar MRI at the consult on the hospital and to reduce visits at the hospital for the patient.

The study relied on data from the newly 2018 update of the NPR at Statistics Denmark. ${ }^{47}$ This allowed for the newest data from all individuals aged $18+$ of the population of Denmark. The granularity of the data allows for an unseen precision of analyses performed on country level.

\section{CONCLUSIONS}

Following RSD's introduction of organisational changes in 2010, the lumbar MRI rate increased significantly in comparison with the other regions in Denmark. The issue of whether the increased usage of lumbar MRI is beneficial for the RSD's patients with LBP (compared with that of other regions) requires further investigations.

\section{Author affiliations}

${ }^{1}$ The Institute of Regional Health Research, University of Southern Denmark, Odense, Denmark

${ }^{2}$ VIVE, The Danish Center of Social Science Research, Copenhagen, UK ${ }^{3} \mathrm{DaCHE}$, Institute of Public Health, University of Southern Denmark, Odense, Denmark

${ }^{4}$ Centre for Quality, Region of Southern Denmark, Middelfart, Denmark ${ }^{5}$ Research Unit of General Practice, Institute of Public Health, University of Southern Denmark, Odense, Denmark

${ }^{6}$ Spine Centre of Southern Denmark, Lillebaelt Hospital, Region of Southern Denmark, Middelfart, Denmark

Contributors MSJ and KRO made the data set at Statistics Denmark, analysed the data, wrote the manuscript, made figures and tables with contributions from LM, JS and BS-C. All authors read and approved the final manuscript.

Funding The study is a part of a three-year PhD grant with contributions from Centre for Quality, Region of Southern Denmark, The Institute of Regional Health 
Research, University of Southern Denmark and Region of Southern Denmark. The Study further received a grant from the Regional Research Fund of Southern Denmark, Region of Southern Denmark. All grant givers had no saying in the design and interpretation of the study.

Competing interests None declared.

Patient consent for publication Not required.

Ethics approval The study is based on registry data, which does not require ethics approval in Denmark (Act on Research Ethics Review of Health Research Projects § 14, sec. 2 http://www.nvk.dk/english/act-on-research10.02.2017). The study group did however send the study protocol for an ethic assessment to the regional ethics committee in Region of Southern Denmark. The committee found that the study did not need ethic approval. The Danish Data Protection Agency approved this study (Journal number 15/14594).

Provenance and peer review Not commissioned; externally peer reviewed.

Data sharing statement The data that support the findings of this study are available from national databases at Statistics Denmark (Sejrøgade 11, DK-2100 København Ø, Phone +45 391731 30, E-Mail forskningsservice@dst.dk), a governmental institution, but restrictions apply to the availability of these data, which were used under license for the current study, and so are not publicly available. Data are, however, available from the authors upon reasonable request and with permission of Statistics Denmark and The Danish Data Protection Agency (Borgergade 28, DK-1300 Copenhagen, Phone +45 3319 3200, E-Mail dt@ datatilsynet.dk).

Open access This is an open access article distributed in accordance with the Creative Commons Attribution Non Commercial (CC BY-NC 4.0) license, which permits others to distribute, remix, adapt, build upon this work non-commercially, and license their derivative works on different terms, provided the original work is properly cited, appropriate credit is given, any changes made indicated, and the use is non-commercial. See: http://creativecommons.org/licenses/by-nc/4.0/.

\section{REFERENCES}

1. Chou R, Qaseem A, Snow V, et al. Diagnosis and treatment of low back pain: a joint clinical practice guideline from the American College of Physicians and the American Pain Society. Ann Intern Med 2007;147:478-91.

2. Dagenais S, Tricco AC, Haldeman S. Synthesis of recommendations for the assessment and management of low back pain from recent clinical practice guidelines. Spine J 2010;10:514-29.

3. Deyo RA, Mirza SK, Turner JA, et al. Overtreating chronic back pain: time to back off? J Am Board Fam Med 2009;22:62-8.

4. Dagenais S, Caro J, Haldeman S. A systematic review of low back pain cost of illness studies in the United States and internationally. Spine J 2008;8:8-20.

5. Lurie JD, Birkmeyer NJ, Weinstein JN. Rates of advanced spinal imaging and spine surgery. Spine 2003;28:616-20.

6. Wang D, Workers Compensation Research Institute (Cambridge Mass). Interstate variations in medical practice patterns for low back conditions. Cambridge, Mass: Workers Compensation Research Institute, 2008.

7. Dagenais S, Galloway EK, Roffey DM. A systematic review of diagnostic imaging use for low back pain in the United States. Spine J 2014;14:1036-48.

8. Mitchell JM. Utilization trends for advanced imaging procedures: evidence from individuals with private insurance coverage in California. Med Care 2008;46:460-6.

9. Mitchell JM. Do financial incentives linked to ownership of specialty hospitals affect physicians' practice patterns? Med Care 2008;46:732-7.

10. Swedlow A, Johnson G, Smithline N, et al. Increased costs and rates of use in the California workers' compensation system as a result of self-referral by physicians. N Engl J Med 1992;327:1502-6.

11. Baras JD, Baker LC. Magnetic resonance imaging and low back pain care for Medicare patients. Health Aff 2009;28:w1133-w1140.

12. Pransky G, Foley G, Cifuentes M, et al. Geographic variation in early mri for acute work-related low back pain and associated factors. Spine 2015;40:1712-8.

13. Akbari A, Mayhew A, Al-Alawi MA, et al. Interventions to improve outpatient referrals from primary care to secondary care. Cochrane Database Syst Rev 2008-CD005471-CD71.

14. Kindrachuk DR, Fourney DR. Spine surgery referrals redirected through a multidisciplinary care pathway: effects of nonsurgeon triage including MRI utilization. J Neurosurg Spine 2014;20:87-92.
15. Foster NE, Anema JR, Cherkin D, et al. Prevention and treatment of low back pain: evidence, challenges, and promising directions. Lancet 2018;391:2368-83.

16. Pedersen KM, Andersen JS, Søndergaard J. General practice and primary health care in Denmark. J Am Board Fam Med 2012;25 Suppl 1(Suppl 1):S34-S38.

17. Register- og variabeloversigter Danmarks Statistik: Danmarks Statistik. 2018 http://www.dst.dk/da/TilSalg/Forskningsservice/Data/ Register_Variabeloversigter.aspx (Accessed 14 May 2018).

18. Pedersen CB. The Danish Civil Registration System. Scand J Public Health 2011;39-22-5.

19. Frank L. When an Entire Country Is a Cohort. Science 2000;287:2398

20. Andersen TF, Madsen M, Jørgensen J, et al. The danish national hospital register. A valuable source of data for modern health sciences. Dan Med Bull 1999;46:263-8.

21. Lynge E, Sandegaard JL, Rebolj M. The danish national patient register. Scand J Public Health 2011;39:30-3.

22. Andersen JS, Olivarius NF, Krasnik $A$. The danish national health service register. Scand J Public Health 2011;39:34-7.

23. Kristensen T, Waldorff FB, Nexøe J, et al. Variation in point-of-care testing of hba1c in diabetes care in general practice. Int $J$ Environ Res Public Health 2017;14:1363.

24. Kildemoes HW, Sørensen HT, Hallas J. The Danish National Prescription Registry. Scand J Public Health 2011;39:38-41.

25. ATC -Structure and principles WHO: WHO Collaborating Centre for Drug Statistics Methodology. 2018. updated 15 Feb 2018 https:// www.whocc.no/atc/structure_and_principles/ (Accessed 14 May 2018).

26. Baadsgaard M, Quitzau J. Danish registers on personal income and transfer payments. Scand J Public Health 2011;39(7 Suppl):103-5.

27. Jensen VM, Rasmussen AW. Danish education registers. Scand J Public Health 2011;39:91-4.

28. Petersson F, Baadsgaard M, Thygesen LC. Danish registers on personal labour market affiliation. Scand J Public Health 2011;39-95-8.

29. Lechner M. The Estimation of Causal Effects by Difference-inDifference Methods. Foundations and Trends ${ }^{\circledR}$ in Econometrics 2011;4:165-224.

30. Rosenbaum PR, Rubin DB. The central role of the propensity score in observational studies for causal effects. Biometrika 1983;70:41-55.

31. Imbens GW, Wooldridge JM. Recent developments in the econometrics of program evaluation. J Econ Lit 2009;47:5-86.

32. Huber M, Lechner M, Wunsch $C$. The performance of estimators based on the propensity score. J Econom 2013;175:1-21.

33. Charlson ME, Pompei P, Ales KL, et al. A new method of classifying prognostic comorbidity in longitudinal studies: development and validation. J Chronic Dis 1987;40:373-83.

34. Arpino B, Cannas M. Propensity score matching with clustered data. An application to the estimation of the impact of caesarean section on the Apgar score. Stat Med 2016;35:2074-91.

35. McPhillips-Tangum CA, Cherkin DC, Rhodes LA, et al. Reasons for repeated medical visits among patients with chronic back pain. $J$ Gen Intern Med 1998;13:289-95.

36. Kendrick D, Fielding K, Bentley $\mathrm{E}$, et al. The role of radiography in primary care patients with low back pain of at least 6 weeks duration: a randomised (unblinded) controlled trial. Health Technol Assess 2001;5:69.

37. Rao JK, Kroenke K, Mihaliak KA, et al. Can guidelines impact the ordering of magnetic resonance imaging studies by primary care providers for low back pain? Am J Manag Care 2002;8:27-35.

38. Gidwani R, Sinnott P, Avoundjian T, et al. Inappropriate ordering of lumbar spine magnetic resonance imaging: are providers Choosing Wisely? Am J Manag Care 2016;22:e68-76.

39. Freeborn DK, Shye D, Mullooly JP, et al. Primary care physicians' use of lumbar spine imaging tests: effects of guidelines and practice pattern feedback. J Gen Intern Med 1997;12:619-25.

40. Webster BS, Bauer AZ, Choi Y, et al. latrogenic consequences of early magnetic resonance imaging in acute, work-related, disabling low back pain. Spine 2013;38:1939-46.

41. Webster BS, Choi Y, Bauer AZ, et al. The cascade of medical services and associated longitudinal costs due to nonadherent magnetic resonance imaging for low back pain. Spine 2014;39:1433-40.

42. Webster BS, Cifuentes M. Relationship of early magnetic resonance imaging for work-related acute low back pain with disability and medical utilization outcomes. J Occup Environ Med 2010;52:900-7.

43. Graves JM, Fulton-Kehoe D, Jarvik JG, et al. Early imaging for acute low back pain: one-year health and disability outcomes among Washington State workers. Spine 2012;37:1617-27. 
44. Graves JM, Fulton-Kehoe D, Jarvik JG, et al. Health care utilization and costs associated with adherence to clinical practice guidelines for early magnetic resonance imaging among workers with acute occupational low back pain. Health Serv Res

2014;49:645-65.

45. Graves JM, Fulton-Kehoe D, Martin DP, et al. Factors associated with early magnetic resonance imaging utilization for acute occupational low back pain: a population-based study from Washington State workers' compensation. Spine 2012;37:1708-18.

46. Chou R, Fu R, Carrino JA, et al. Imaging strategies for low-back pain: systematic review and meta-analysis. Lancet 2009;373:463-72.

47. Nyt fra Forskerservice nr. 4/2017 Forskerservice: Danmarks Statisitk. 2017;4. cited 14 May 2018 https://www.dst.dk/da/TilSalg/ Forskningsservice/Meddelelser. 